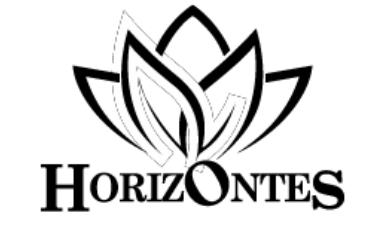

www.revistahorizontes.org
Horizontes. Revista de Investigación en Ciencias de la Educación Https://doi.org/10.33996/revistahorizontes.v4i15.108 Julio - Septiembre 2020

Volumen 4 / No. 15 ISSN: $2616-7964$ ISSN-L: $2616-7964$ pp. $181-194$

\title{
Competencias docentes en los Institutos Universitarios de Tecnología Públicos del Estado Zulia
}

\author{
Teaching academic competencies in the University Institutes of Public Technology of the \\ State Zulia
}

\section{Competências de ensino nos Institutos Universitários Públicos de Tecnologia do Estado de Zulia}

ARTICULO ORIGINAL

\author{
Gabriel Belgrave \\ gbelgrave65@hotmail.com
}

Universidad Politécnica Territorial del Zulia, Venezuela

Recibido marzo 2020 | Revisado abril 2020 | Publicado en julio 2020

\section{RESUMEN}

La formación del docente universitario representa un desafío ante un contexto social dominado por los continuos e inesperados cambios generados por la globalización. Esto implica reorientar el proceso educativo hacia senderos de excelencia, a través de la preparación, capacitación y formación de los docentes, quienes dan vida a esta labor, como lo es formar las generaciones de relevo que la sociedad reclama. La finalidad del estudio consistió en indagar sobre la formación de los docentes basada y enfocada en las competencias para asumir nuevas oportunidades que les permitan mejorar la manera de producir, organizar, difundir y acceder al saber $\mathrm{y}$ al conocimiento, manteniendo como objetivo el describir las competencias docentes en los Institutos Universitarios de Tecnología (IUT) públicos del estado Zulia. La investigación fue de tipo descriptiva con un diseño de campo, transeccional contemporánea, con una amplitud de foco un eventual. Los resultados revelaron que los docentes presentaban debilidades en las competencias comunicacionales y competencias técnicas.

Key words: Competencias, perfil por competencias, competencias de comunicación y competencias técnicas

\section{ABSTRACT}

University teacher training represents a challenge in a social context dominated by the continuous and unexpected changes generated by globalization. This implies reorienting the educational process towards paths of excellence, through the preparation, training and education of teachers, who give life to this work, such as training the successor generations that society demands. The purpose of the study was to inquire about the training of teachers based and focused on competencies to take on new opportunities that allow them to improve the way of producing, organizing, disseminating and accessing knowledge and knowledge, keeping the objective of describing competences teachers at the public University Institutes of Technology (IUT) of the state of Zulia. The research was descriptive with a contemporary, cross-sectional field design, with an eventual breadth of focus. The results revealed that teachers had weaknesses in communication skills and technical skills.

Palabras clave: Competences, profile by competences, communication skills and technical skills 


\section{RESUMO}

A formação de professores universitários representa um desafio em um contexto social dominado pelas mudanças contínuas e inesperadas geradas pela globalização. Isso implica reorientar o processo educacional em direção a caminhos de excelência, por meio da preparação, treinamento e formação de professores, que dão vida a esse trabalho, como o treinamento das gerações sucessoras que a sociedade exige. 0 objetivo do estudo foi investigar a formação de professores com base e focada em competências para aproveitar novas oportunidades que lhes permitam melhorar a maneira de produzir, organizar, disseminar e acessar conhecimentos e conhecimentos, mantendo o objetivo de descrever competências. Professores dos Institutos Universitários de Tecnologia (IUT) públicos do estado de Zulia. A pesquisa foi descritiva, com um design de campo contemporâneo e transversal, com uma eventual amplitude de foco. Os resultados revelaram que os professores apresentaram fragilidades nas habilidades de comunicação e técnicas.

Palavras-Chave: Competências, perfil por competências, habilidades de comunicação e habilidades técnicas

\section{INTRODUCCIÓN}

Frente a los retos que genera un entorno globalizado y cambiante como el actual, las organizaciones están en la obligación de buscar estrategias centradas en el quehacer de sus trabajadores, que les permita sustentar la estabilidad y crecimiento exitoso, ante esto, es menester que el individuo desarrolle habilidades y destrezas que le permitan potenciar sus competencias, a fin de formar redes de trabajo encaminadas a alcanzar metas comunes.

A mediado de la década de 1960, países como Inglaterra y Alemania consideraban que las personas que aspiraban a desempeñar un oficio debían tener conocimientos especiales, que aplicados correctamente les facultaba para desempeñarse perfectamente en un oficio. Luego, a finales de los años 1970 se implementan sistemas de formación para el trabajo basados en criterios de calidad y eficiencia, en el marco de la competitividad que arropaba a los países desarrollados. Así, las exigencias de las organizaciones respecto al mejoramiento del desempeño del trabajador se han mantenido en el tiempo, pues, tal como lo refieren Luthans y Youssef (2004), la productividad y eficiencia que puede alcanzar una organización está en función de la productividad y eficiencia de sus trabajadores.

De igual manera, hoy la sociedad reclama la formación de un ciudadano crítico, instruido culturalmente y competente para desenvolverse efectividad en su área de trabajo, así como ante las adversidades que presenta la vida, por lo cual es necesario desarrollar vínculos entre el sector formador del capital humano y las organizaciones, es decir formar recursos humanos donde el énfasis este centrado en la eficiencia, la equidad y la eficacia; tal como lo enuncia Jurado (2003 c.p. Tobón 2006, p. 41) “...esta tendencia la apoya e impulsa el Banco Mundial, y plantea la necesidad de que las instituciones educativas formen el capital humano que requiere el mercado local y global". De igual manera Sen, Zamagni $y$ Scazzieri (2008 p. 45) afirma que "la riqueza de un país está íntimamente ligada al nivel educativo alcanzado por una sociedad o nación, por lo cual en la búsqueda del desarrollo necesario, la pertinencia de la educación ocupa un rol protagónico".

A partir de lo mencionado, puede afirmarse que todos los sistemas educativos están ganados a trabajar en mejoras de la educación y por ende, en la formación de sus ciudadanos; sin embargo, ello constituye una tarea ardua y por demás compleja, puesto que en ella están inmersos múltiples factores tales

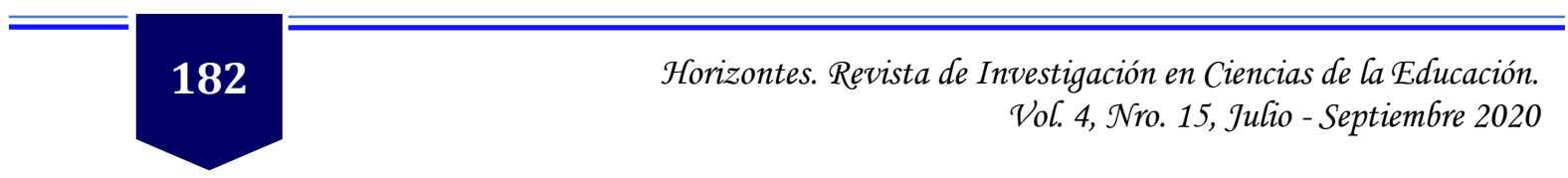


como las políticas de estado, el modelo pedagógico adoptado, la gestión de las instituciones, el rol de la sociedad como coparticipe del hecho educativo, entre otros.

Una revisión histórica de lo que ha significado la docencia en Latinoamérica desde inicios del siglo XIX hasta la actualidad, evidencia que la misma es una profesión fundamentalmente social razón por la cual depende de todos los factores contextuales presentes en el momento histórico donde se desarrolla, así como de las fuerzas que actúan en la sociedad e inciden particularmente sobre el estado, orientando las decisiones de política educativa; sin embargo, una constante es que las instituciones formadoras necesitan producir profesionales competentes para atender los desafíos de las sociedades que imperen en el momento histórico que les toque vivir; lo que pone de manifiesto el papel que juega el rol docente como elemento determinante de la calidad de la educación alcanzada en cada país o continente.

En Venezuela, las instituciones de educación superior, y particularmente del estado Zulia, como entes formadores de recurso humano calificado, no escapan de estos retos por lo que cada vez más se enfatiza el debate acerca de los mecanismos que deben utilizar para formar los recursos humanos requeridos, así como la necesidad de revisión de los contenidos programáticos, los métodos de enseñanza y muy particularmente del perfil del docente; para generar cambios y transformaciones en la manera de enseñar en todos los niveles del sistema educativo, que sean cónsonos con las exigencias de la sociedad actual.

De igual manera, la Ley Orgánica de Educación (2009) y la Ley de Universidades (1970), ambos documentos venezolanos, plantean que en las instituciones de educación superior reposa la responsabilidad de colaborar en la orientación de la vida del país, mediante su contribución doctrinaria en el esclarecimiento de los problemas nacionales, además, cumplen una función rectora en la educación, la cultura y la ciencia; asimismo, corresponde a éstas la formación de los profesionales y técnicos que requiere el país para su desarrollo y progreso.

En razón de lo planteado, las instituciones de educación superior venezolanas deben desarrollar procesos de gestión orientados al fortalecimiento de su diario quehacer, que apoye el mantenimiento de la autonomía institucional en el marco de las políticas públicas, así como el enriquecimiento de los procesos pedagógicos, con el fin de responder a las necesidades educativas locales, regionales y nacionales.

Es importante señalar que en cada uno de los retos que afronta el sistema educativo venezolano el docente se destaca como un factor clave para alcanzar las metas establecidas, razón por la cual se hace medular su constante capacitación a fin de que se mantenga en consonancia con las novedades que se presentan en el mundo, postura que es sostenida por la UNESCO (2000, s/p) al aseverar: "El futuro de las organizaciones, y en particular de la educación, dependerá de su capacidad para formar adecuadamente a sus elencos, tanto docentes como administrativos".

Es importante señalar que la búsqueda de niveles óptimos de desarrollo en todos los ámbitos, aunada a la aceleración del cambio social en el momento actual a nivel mundial, exige sucesivos esfuerzos de renovación en el trabajo cotidiano de los docentes.

Desde esa perspectiva, se entiende que la labor docente no se refiere a la simple transmisión de contenidos, sino que requiere el desarrollo de habilidades, actitudes y valores complejos, con la perspectiva de 
provocar el desarrollo de competencias en los educandos. En este orden de ideas, Alanís (2004 p. 18) plantea que un docente debe ser "un profesional de la educación que sea simultáneamente creativo, responsable, gestor de proyectos, que investigue y genere los conocimientos que aplique en la docencia".

De igual manera, Segura (2004 p. 22) plantea que el docente debe asumir el cambio como parte de su ser y quehacer... creando y recreándose en ambientes que tendrán los atributos diversos relacionados con su conocimiento y destrezas... destrezas gerenciales e innovación y creatividad", es decir, un individuo con altos niveles de competencias.

En razón de lo planteado por Zabala y Arnau (2008 p. 43), define las competencias como "La capacidad o habilidad de hacer tareas o hacer frente a situaciones diversas de forma eficaz en un contexto determinado y para ello es necesario movilizar actitudes, habilidades y conocimiento al mismo tiempo y de forma interrelacionada". En toda competencia se conjugan, simultáneamente, conocimientos, habilidades, valores $\mathrm{y}$ responsabilidades para asumir los resultados de lo realizado (Ropé y Tanguy 1994).

Así, al visualizar al docente universitario que exige la actual sociedad, debe pensarse entonces, en un individuo capaz de enfrentar retos y adversidades, con sólidos conocimientos, responsabilidad y ética, que se constituirán en bastiones para asumir de manera airosa las nuevas demandas del saber.

Sin embargo, a pesar de lo anteriormente señalado, autores Luque y Mujica de López (2006), Uricare (2010), Villarroel (1998), Aguerrondo (2000), en sus investigaciones señalan una serie de debilidades presentes en el desempeño del docente universitario venezolano, tales como: actitud pasiva $\mathrm{y}$ acomodaticia frente a los inconvenientes que desde muchos años envuelve su formación y desarrollo profesional, irrestricto apego a la pedagogía tradicional por lo que concibe al estudiante como un ente pasivo, desvinculación entre la formación teórica y la formación práctica, escaso manejo de las tecnologías de la información y la comunicación, existencia de dominación y control sobre los estudiantes, entre otros.

Lo anteriormente descrito ha traído como consecuencia que, aunque en teoría el perfil docente predominante es considerado apropiado para ocupar un cargo universitario, el desempeño de las funciones docentes presente grandes vacíos.

Como muestra de la problemática descrita, se encuentran los Institutos Universitarios de Tecnología (IUT) públicos ubicados en el Estado Zulia, los cuales forman parte del subsistema de la educación universitaria en Venezuela. Estas instituciones de educación superior fueron creados, según el Reglamento de los Institutos y Colegios Universitarios (1995), con el objeto, fundamentalmente, de formar individuos con una sólida actitud crítica ante los problemas de la sociedad contemporánea, así como suministrarles los instrumentos conceptuales básicos que les permita afrontar con éxito los futuros retos profesionales que la dinámica socioeconómica les exija.

Para el logro de estos objetivos, el estado venezolano ha realizado importantes reformas en las políticas educativas implementadas a nivel nacional, tales como la creación de la Universidad Bolivariana de Venezuela (UBV), la Misión Sucre, la constitución de la Misión Alma Mater $\mathrm{y}$, más recientemente, con la creación de las nuevas Universidades Politécnicas Territoriales (UPT) a partir de la transformación de los institutos y colegios universitarios (IUT y CU). 
Por otra parte, la Ley del Plan de la Patria - Segundo Plan Socialista de Desarrollo Económico y Social de la Nación 2013-2019, plasma la necesidad de enfocar los esfuerzos en desarrollar las capacidades científico tecnológicas vinculadas a las necesidades territoriales, lo que implica la consolidación del carácter transformador de la educación universitaria, el aprovechamiento de las potencialidades de los IUT, creación y recreación de saberes, el impulso a la creación intelectual e innovación que responda a los problemas prioritarios del país, al trabajo productivo, así como a los objetivos estratégicos del plan nacional.

En efecto, a partir de año 2009, los IUT públicos se pliegan a este gran reto a través de la implementación de un nuevo modelo curricular: los Programas Nacionales de Formación (PNF), cuyos objetivos persiguen fortalecer e incentivar la investigación en el proceso educativo, permitiendo el acceso al conocimiento para universalizar la educación superior, así como garantizar la pertinencia. Dichos programas requieren, a la vez, que los docentes de estas instituciones estén a la par de estos retos.

Sin embargo, de acuerdo con el Ministerio del Poder Popular para la Educación Universitaria, Ciencia y Tecnología (2015), la puesta en marcha de los PNF no ha sido suficiente puesto que ha generado la necesidad de una transformación fundamental en el perfil del docente y sus competencias, para asumir las funciones de formación integral, creación intelectual e integración socio comunitaria y socio productiva en un marco de nuevas relaciones.

En razón de la problemática planteada, es menester conocer a profundidad las competencias que conforman el perfil del docente, ante lo cual surge la pregunta de investigación ¿Cuáles son las competencias docentes en los Institutos Universitarios de Tecnología públicos del estado Zulia?

\section{Aspectos teóricos}

En Venezuela, la educación se ha caracterizado por una serie de reformas, las cuales se han incrementado en los últimos años. Todos estos cambios han tenido como intencionalidad lograr la ruptura de los modelos curriculares y didácticos tradicionales, impulsando nuevos paradigmas que apoyen la construcción de nuevos modelos curriculares donde se conjuguen criterios, tales como: mayor equidad en el acceso a la educación, mejoras en la calidad del servicio ofrecido, así como de sus resultados. Estas reformas se han visto plasmadas a través de diferentes proyectos o enfoques sobre la práctica pedagógica.

El Proyecto Tuning América Latina nace en el año 2000 a raíz de las necesidades de reconocimiento mutuo de los periodos de estudio en el extranjero y de los títulos de grado que emergieron con la Declaración de Bologna de 1999. Dicha declaración establecía que para el año 2010 debía existir una educación superior coherente, compatible y competitiva, centrado en la importancia del desarrollo curricular, la cooperación entre las instituciones, los esquemas de movilidad y la integración de la formación e investigación.

El diseño curricular bajo el enfoque de competencias profesionales tiene sus orígenes en la Declaración de Bolonia de 1999 y el cual fue asumido en varios países de América Latina. En Venezuela sólo algunas instituciones de educación superior implementaron el diseño curricular bajo el enfoque de competencias.

\section{Competencias}

En el devenir del tiempo el término competencia ha sido definido bajo diferentes 
enfoques por diversos autores, esto ha llevado a algunos autores a proponer el término "movimiento de las competencias" para incluir a toda la variedad de definiciones. Se considera que fue McClellan (1973) quien introdujo por vez primera en el mundo laboral, el concepto de competencia. Para este autor era de gran relevancia considerar las características del trabajador y su actuación ante eventos concretos, puesto que, a su criterio, de éstas dependían las competencias que desempeñaba en el trabajo.

Por su parte, Reis (1994 c.p. Mertens, 1996, p. 62) señala que la competencia es la "capacidad real del individuo para dominar el conjunto de tareas que configuran la función en concreto".

De igual manera, Tobón (2008), define competencias como:

Procesos complejos de desempeño con idoneidad en determinados contextos, integrando diferentes saberes (saber ser, saber hacer, saber conocer y saber convivir), para realizar actividades y/o resolver problemas con sentido de reto, motivación, flexibilidad, creatividad, comprensión y emprendimiento, dentro de una perspectiva de procesamiento metacognitivo, mejoramiento continuo y compromiso ético, con la meta de contribuir al desarrollo personal, la construcción y afianzamiento del tejido social, la búsqueda continua del desarrollo económico-empresarial sostenible, y el cuidado y protección del ambiente y de las especies vivas (p. 5)

Así mismo, Boyatzis (1982 c.p. Hoffmann, 1999, p. 278) define la competencia como "una característica subyacente de una persona que da por resultado un desempeño efectivo y/o superior en un cargo"; en tanto que para Mertens (1996 p. 61), una competencia es la "capacidad real para lograr un objetivo o resultado en un contexto dado".
En el mismo orden de ideas, Ansorena (1996, p. 76) plantea: "Una habilidad o atributo personal de la conducta de un sujeto, que puede definirse como característica de su comportamiento, y, bajo la cual, el comportamiento orientado a la tarea puede clasificarse de forma lógica y fiable."

Resulta interesante resaltar que aun cuando los autores sustentan diferentes definiciones, todos coinciden en que la competencia es la capacidad de realizar una acción concreta, al disponer simultáneamente del conjunto de conocimientos necesarios para su desarrollo (saber), las habilidades o destrezas requeridas para realizarla (saber hacer) y la actitud orientada a su realización y la obtención de un resultado eficiente (querer hacer y hacer eficientemente). De hecho, las competencias combinan en sí, algo que los constructos psicológicos tienden a separar (a sabiendas de la artificialidad de la separación): lo cognoscitivo (conocimientos y habilidades), lo afectivo (motivaciones, actitudes, rasgos de personalidad), lo psicomotriz o conductual (hábitos, destrezas) y lo psicofísico o psicofisiológico.

En efecto, una competencia es lo que hace que la persona tenga la capacidad para realizar un trabajo o una actividad y sea exitoso en la misma, lo que puede significar la conjunción de conocimientos, habilidades, disposiciones y conductas específicas. Si falla alguno de esos aspectos, y el mismo se requiere para lograr algo, entonces, ya no se es "competente".

De igual manera, al revisar las características o componentes de las competencias, se observa que, de alguna manera, están asociados con los constructos psicológicos los cuales se combinan de una manera determinada para generar la capacidad de rendir eficientemente en tareas o actividades 
específicas, es decir, dichas combinaciones hacen a la persona "competente". Obviamente, determinar la forma en que se combinan dichos constructos sólo es posible mediante el análisis del comportamiento que asumen en el trabajo las personas exitosas.

El número de competencias existentes puede ser muy amplio: Levy-Leboyer (2003) presenta seis diferentes listas, Ansorena (1996) incluye 50 competencias conductuales, Scriven (1997), presenta 8 listas de competencias, por citar algunos. Así mismo, Tobón (2006), considera que existen al menos cuatro enfoques a través de los cuales se puede analizar las competencias, los cuales deben ser considerados para su comprensión, estos son: el conductista u ocupacional, el funcionalista, el constructivista y el holístico. Siendo el enfoque constructivista el que más se adapta al campo educativo por concordar con la idea sobre el carácter constructivista que presenta el conocimiento.

Si bien es cierto que en los programas educativos el interés en el desarrollo de competencias concuerda con un enfoque de la educación centrado ante todo en el alumno y en su capacidad de aprender, lo cual exige más protagonismo y cuotas más altas de compromiso, ya que es el estudiante quien debe desarrollar las capacidades, las habilidades y las destrezas, de igual forma es necesario determinar qué elementos cognitivos, actitudinales, valorativos $\mathrm{y}$ de destrezas favorecen la resolución de los problemas educativos, desde todos los niveles de desempeño del docente, es decir, es medular delinear el perfil por competencias requerido por el docente para garantizar la formación del nuevo hombre reclamado por la actual sociedad.

Establecer el perfil del docente va más allá de un simple listado de competencias, es preciso indagar minuciosamente sobre las habilidades y conocimientos requeridos, así como los comportamientos y actitudes necesarias para desempeñarse exitosamente en el puesto de trabajo, por lo que los perfiles de competencias de cargos superan a los tradicionales perfiles de cargo o profesiogramas que comprenden funciones descritas en un plano netamente cognitivo.

Según Alles (s/f) un perfil por competencias es un modelo integral de gestión por competencias donde se declaran las diferentes competencias y los niveles o grados requeridos para cada una de ellas. Por lo general, un perfil por competencias o modelo integral de gestión por competencias presenta dos categorías de competencias: competencias genéricas $\mathrm{o}$ básicas $\mathrm{y}$ competencias técnicas o específicas.

En este mismo orden de ideas, AQU (2002, c.p. Cano, 2005) considera que las competencias genéricas o básicas son aquellas que se consideran esenciales para el desarrollo vital de todos los individuos, y hacen referencia a saberes transversales asociados a desempeños que se ponen de manifiesto de manera habituales.

A su vez, las competencias técnicas o específicas se derivan de las exigencias de un contexto o trabajo concreto. Son competencias propias o vinculadas a una titulación y que proporcionan identidad y consistencia social y profesional al perfil formativo. A su vez, pueden dividirse también según los siguientes ámbitos: las referidas al conocimiento, las profesionales, y las académicas.

Según Bunk (1994), las competencias profesionales son un conjunto de conocimientos, destrezas y aptitudes que se necesitan al ejercer una profesión, resolver problemas de la misma profesión autónoma y flexiblemente, así como, tener la capacidad de desarrollar lo referido al contexto profesional y organización del trabajo. Este autor 
considera una tipología de las competencias profesionales, siendo estas: técnicas, metodológicas, sociales y participativas, en donde cada una tiene una especificación. Finalmente, al integrarse como un todo estas cuatro competencias parciales surge la competencia de acción, que es totalmente indivisible.

Por su parte, Ducci (1997) considera la competencia profesional como la construcción social de aprendizajes significativos y válidos para el desempeño laboral en una situación real de trabajo que se obtiene no sólo a través de la instrucción, sino también, y en gran proporción, a través del aprendizaje por experiencia en situaciones concretas de trabajo.

Asimismo, Epstein y Hundert (2002 c.p. Miyahira 2007), definen competencia profesional como:

el uso habitual y juicioso de la comunicación, el conocimiento, las habilidades, las técnicas, el razonamiento, las emociones, los valores y la reflexión en la práctica cotidiana para el beneficio de la persona y la comunidad a la que está ofreciendo su servicio (p. 1)

De lo antes planteado, se infiere que las competencias profesionales se plantean en un contexto cambiante; que se mantienen en constante evolución y caracterizan a un profesional competente que se hace cargo de una situación concreta a través de operaciones múltiples y que éstas se determinan a través de criterios y comportamientos observables por lo cual la evaluación del desempeño se constituye en parte fundamental de las mismas competencias.

\section{Competencias específicas en el ámbito académico}

Una de las profesiones en las que más ha incidido la utilización del concepto competencia, sobre todo en relación con la formación, es la profesión docente. Esto ha llevado a plantear las siguientes interrogantes: ¿cuál es el profesor necesario para garantizar a todos los alumnados una apropiada educación que se traduzca en una óptima calidad de los aprendizajes adquiridos? Además, ¿qué competencias y estándares en cada especialidad se requieren para movilizar conocimientos, capacidades, estrategias innovadoras y efectivas para el proceso de enseñanza y aprendizaje?

La formación propiciada por las instituciones educativas, en este caso, las universidades, debe traspasar la frontera de la simple incorporación del sujeto a la vida productiva a través del empleo, dicha formación profesional debe, además de promover el desarrollo de ciertos atributos (habilidades, conocimientos, actitudes, aptitudes y valores), modificar el pensamiento del individuo de manera que enfrente la complejidad creciente, la rapidez de los cambios y lo imprevisible del mundo de hoy, (Morín, 2000).

La complejidad de lo planteado, la contextualización y exigencias, idiosincrasia y cultura de cada región ha permitido la aparición de múltiples propuestas de competencias docentes, tales como las planteadas por Leboyer (2003); Perrenoud (2004); Cano (2005); Pavie (2009), Angulo (1999 c.p. Cano 2005), por citar algunos.

Angulo (1999), en su proceso de selección de competencias docentes considera las cinco clases de competencias propuestas por Houston en 1980, es importante acotar que, en este proceso, dicho autor no adopta un modelo de entrenamiento previo, sino, más bien, considera que la práctica docente es el procedimiento idóneo de adquisición de dichas competencias. Tomando en cuenta que los elementos que incluyen las competencias docentes deben facilitar el aprendizaje de los estudiantes y además, crear un clima armónico con el 
alumnado, Angulo (1999) declara la siguiente clasificación de competencias docentes: destrezas de comunicación, conocimientos básicos, destrezas técnicas, destrezas administrativas y destrezas interpersonales.

A su vez, Scriven (1997) presenta un listado de competencias académicas a las cuales considera el buen hacer de un docente, siendo estas: conocimiento de la responsabilidad de la enseñanza, planificación y organización de la enseñanza, comunicación, organización de la clase, eficacia en la instrucción, evaluación, profesionalidad y otros servicios individualizados al centro y a su comunidad.

Sin embargo, todo listado y definiciones sobre competencias quedan sin efecto si previamente el docente no asume su rol fundamental, el de ser el guía para que cada educando, incluido el mismo, encuentre su propio camino hacia el desarrollo integral de la personalidad y por ende el desarrollo de toda la comunidad, así, el docente de hoy, como formador del hombre del mañana, tiene la misión de perfilar situaciones problemáticas dirigidas a fomentar la capacidad de sus alumnos para tomar decisiones y responder por sus actos; es decir, lograr la autonomía en el estudiantado lo cual se inicia al ser considerado sujeto de la educación y no objeto de la misma.

En efecto, lo antes planteado es reafirmado por Delors (1997) cuando considera que:

El trabajo del docente consiste no sólo en transmitir información ni siquiera conocimientos, sino en presentarlos en forma de problemática, situándolos en un contexto y poniendo los problemas en perspectiva, de manera que el alumno pueda establecer el nexo entre su solución y otros interrogantes de mayor alcance. (p. 164)
De igual manera, es cierto que si el docente conoce cuáles competencias se esperan del educando al finalizar una etapa dentro del programa educativo, podrá dirigirlos en esa dirección, lo cual implica a su vez que el educador debe aprender a aprender, de manera tal que se vea a sí mismo como una construcción continua y permanente, ya que la producción de conocimientos es constante y cada vez más acelerada y cambiante (Frade, 2009).

La sociedad contemporánea exige mejores logros en la ejecución de la labor docente, en razón de lo cual se asume la postura de Farías (2008) quien sostiene que "es imperante que nuestros sistemas educativos se adecúen a los nuevos tiempos y más imprescindible que las instituciones formadoras de docentes entiendan que es tarea primordial renovar los viejos pensum de estudio que se han enquistado en ellas" (p.71). puesto que parafraseando a Morín (1999) se trata de "formar individuos con la cabeza bien puesta y no bien llena".

\section{METODOLOGÍA}

La investigación se consideró de tipo descriptiva, con un diseño de campo, no experimental, transeccional. Las técnicas de recolección de datos utilizadas fue la encuesta y como instrumento un cuestionario estructurado, compuesto de 48 ítems de alternativas múltiples. Dicho instrumento fue validado a través del juicio de diez (10) expertos. La confiabilidad se determinó a través del software de aplicación estadística SPSS 18.0 para Windows, resultando un coeficiente de Alfa Crombach de 0,995, lo cual indica que el instrumento era altanamente confiable. La población estuvo conformada por un total de 39 docentes, discriminados de 
la siguiente manera: 16 docentes pertenecientes al Instituto Universitario de Tecnología Cabimas Extensión Ciudad Ojeda (IUTC Ext. Ciudad Ojeda) y 23 pertenecientes al Instituto Universitario de Tecnología Maracaibo (IUTM). Dichos docentes debían reunir las siguientes características: personal docente de carácter ordinario, que labore actualmente dictando clases en el último semestre en el Programa Nacional de
Formación (PNF) en Administración de Empresas, sedes del IUTC Ext Ciudad Ojeda y IUTM. Para el análisis de la información obtenida se emplearon como técnicas de tratamiento de datos la estadística descriptiva, específicamente la medida de tendencia central media, cuyo baremo de medición para la interpretación de la media se especifica en la tabla 1 .

Tabla 1. Categoría de análisis para la interpretación del promedio

$\begin{array}{lll}\text { Intervalo } & \text { Categorías } & \text { Descripción } \\ 4,20-5,00 & \text { Siempre } & \text { Muy altas competencias } \\ 3,40-4,19 & \text { Casi siempre } & \text { Altas competencias } \\ 2,60-3,39 & \text { A veces } & \text { Medianas competencias } \\ 1,80-2,59 & \text { Casi nunca } & \text { Bajas competencias } \\ 1,00-1,79 & \text { Nunca } & \text { Muy baja competencias }\end{array}$

Fuente: Belgrave, (2018)

\section{RESULTADOS Y DISCUSIÓN}

Los resultados referidos a la dimensión competencias profesionales se presentan en la tabla 2, puede observarse que dicha dimensión arrojó una media de 3,21, que al ser comparada con el baremo de interpretación del promedio (tabla 1) se ubicó en la categoría de medianas competencias, lo cual pone de manifiesto una mediana gestión en la labor que desarrollan los decentes adscritos a los Programas Nacionales de Formación (PNF) en la especialidad de Administración de Empresas de los IUTC Ext. Ciudad Ojeda y IUTM.

Tabla 2. Competencias Profesionales

\begin{tabular}{ccc|} 
Competencias & Promedio= $\mathbf{X}$ & Categoría \\
Comunicación & 3,22 & Medianas competencias \\
Técnicas & 2,45 & Bajas competencias \\
Conocimientos básicos & 3,58 & Altas competencias \\
Administrativas & 3,6 & Altas competencias \\
Total & $\mathbf{3 , 2 1}$ & Medianas competencias \\
\hline
\end{tabular}

Fuente: Belgrave, (2018) 
De igual manera, puede observarse en la tabla 2, que los indicadores denominados competencias técnicas, propias de la profesión docente, alcanzó una media de 2,45 lo que según el baremo se ubicó en una categoría de baja competencia, en tanto que comunicación alcanzó una media 3,22 lo cual le ubicó en una categoría de mediana competencias. Comportamiento contrario manifestaron los indicadores conocimientos básicos y administrativos con medias de 3,58 y 3,6 , respectivamente, lo que les ubicó en una categoría de altas competencias.

Sin embargo, se destacan dos debilidades en la gestión de los docentes de las instituciones objeto de estudio, como son la comunicación y las técnicas. Estas competencias son de suma importancia puesto que las competencias referidas a la comunicación permiten a los docentes expresar de manera asertiva a sus estudiantes las ideas, los planteamientos y argumentos sobre el desarrollo de las actividades curriculares, así como brindarles el apoyo integral en su proceso de formación. Asimismo, es menester que el docente alcance una comunicación eficacia, que desarrolle la habilidad y destreza de comunicar escrito y oralmente información sobre tópicos de interés de forma coherente y lógica en sus clases.

Con relación a las competencias técnicas, las cuales según Angulo (1999 c.p. Cano 2005) están referidas a:

a). Diagnosticar los conocimientos y destrezas previas de los estudiantes necesarias para lograr un conjunto de objetivos de enseñanza. b). Identificar objetivos a largo plazo para una materia dada. c). Construir y ordenar secuencialmente objetivos relacionados entre sí a corto plazo. d). Seleccionar, adaptar y elaborar materiales de enseñanza para los objetivos de instrucción y para las necesidades de aprendizaje del alumnado. e). Seleccionar, desarrollar y elaborar secuencias de actividades de aprendizaje relacionadas entre sí, apropiadas para un conjunto dado de objetivos de enseñanza y necesidades de aprendizaje del alumnado. f). Entablar relación con los estudiantes en la clase utilizando recursos motivacionales, verbales y/o visuales. g). Mostrar direcciones para llevar a cabo una actividad instructiva. h). Construir un test para medir el rendimiento del alumnado, de acuerdo con criterios basados en los objetivos (p. 24).

Estas competencias relacionadas con el diario quehacer del docente constituyen las competencias pedagógicas, las cuales son medulares para lograr un desempeño exitoso.

Los resultados obtenidos por la dimensión competencias profesionales son opuestos a lo planteado por Epstein y Hundert (2002 c.p. Miyahira (2007), quienes consideran que las competencias profesionales involucran el uso habitual y juicioso de la comunicación, los conocimientos, las habilidades y destrezas, las técnicas, el razonamiento, las emociones, los valores y la reflexión en el quehacer diario para el beneficio de la persona y la comunidad a la que está ofreciendo su servicio.

Situaciones como las planteadas en las referidas instituciones de educación superior resultan paradójicas, puesto que resulta inexplicable que un docente a pesar de tener altas competencias básicas y administrativas presente medianas competencias en el área de comunicación y bajas competencias en el área técnica, ahora bien, la educación basada en competencias deriva de un enfoque sistémico del proceso de enseñanzaaprendizaje por lo cual, lógicamente, las debilidades antes referidas inciden de forma negativa en el proceso de enseñanza del alumnado, como en la imagen y prestigio de las instituciones de educación superior. 
La educación centrada en competencias exige una constante actualización del docente, lo que implica que el profesional debe ser capaz de reconocer lo que realmente sabe, así como lo que desconoce a manera de planificar estrategias que le lleven a la consecución de los objetivos que le abran espacios para formarse y ser más competente, pero que a la vez estos objetivos logrados se conviertan en incentivos para alcanza nuevas metas de desarrollo a lo largo de su carrera.

Obviamente el proceso de actualización del docente es una tarea compleja, puesto que se bifurca en dos líneas: Por un lado, se requiere que el docente, a través de un aprendizaje autorregulado y en forma de espiral (Polya 1969 c.p. Trías 2008), desarrolle habilidades metacognitivas, las cuales implican un monitoreo en su actuación profesional; además, busque de manera consciente e intencionada la información necesaria, desarrolle las habilidades pertinentes y las lleve a la práctica, para luego reiniciar el ciclo de monitoreo.

Esta forma de abordar el aprendizaje permite al individuo reactivar sus conocimientos previos, con los que compara hechos parecidos, o contrastantes y establece una especie de hipótesis que debe verificar. La situación vuelve a presentarse, sea porque él mismo lo provoca, o porque se da incidentalmente y puede entonces detectar, comparar, criticar, analizar, crear, modificar, corregir o precisar sus saberes.

Por otro lado, las instituciones de educación superior deben prestan especial atención a los factores que afectan el proceso de actualización del docente, tales como escasos o nulos planes de formación y capacitación, poca importancia por parte los entes directivos sobre los beneficios que generan a su personal los proceso de formación, así como la posición de prestigio en la que se posicionan las instituciones al contar con personal docente con amplios méritos académicos, entre otros. En razón de lo planteado, las instituciones de educación superior deben proporcionar a los docentes con competencias y comprometidos con su labor el apoyo que dé muestras sobre la valía de su trabajo y reconocerles que su dedicación garantiza la calidad en la enseñanza superior en las futuras generaciones.

\section{CONCLUSIONES}

En razón de los resultados obtenidos en el estudio de las competencias docentes en los Institutos Universitarios de Tecnología (IUT) públicos del estado Zulia se concluye lo siguiente:

Con relación a la dimensión competencias profesionales, se puso de manifiesto una mediana gestión en la labor que desarrollan los docentes adscritos a los Programas Nacionales de Formación (PNF) en la especialidad de Administración de Empresas de los IUTC Ext. Ciudad Ojeda y IUTM. Se destacó su desempeño en relación a los indicadores referidos a conocimientos básicos $\mathrm{y}$ administrativos, los cuales se ubicaron en altas competencias.

En cuanto al indicador comunicación, los resultados mostraron que los docentes presentan medianas competencias. Este comportamiento llama sumamente la atención ya que para un docente es medular desarrollar una comunicación asertiva con sus estudiantes, puesto que la efectividad del proceso de enseñanza-aprendizaje está influenciada por la comunicación.

De igual manera, resulta preocupante lo referido el bajo nivel alcanzado por las competencias técnicas, ya que están referidas a las habilidades específicas puestas de manifiesto por el docente en el correcto desempeño su área específica. 
Aspectos como los señalados deben ser corregidos por los docentes, bien sea a modo propio o a través de planes de desarrollo gestionados por las instituciones a las cuales están adscritos, pues de ello depende la calidad de su quehacer pedagógico, el desempeño de los estudiantes $\mathrm{y}$, por ende, la imagen de las instituciones.

\section{REFERENCIAS}

Alanís, A. (2004). El Saber Hacer en la Profesión Docente. Formación Profesional en la Práctica Docente. Editorial Trillas, S. A. México

Alles, M. (s/f) Perfil del puesto por competencias. Sepa cómo construirlo y evitar bajos desempeños posteriores. http://www.ucipfg.com/Repositorio/MAP /MAPD-07/UNIDADES-APRENDIZAJE/ UNIDAD_2/perfil_de_puestos_por_compete ncias.pdf

Angulo, (1999) Conversión de las organizaciones educativas en organizaciones que aprenden. Liderazgo y organizaciones que aprenden. ISBN 84271-2338-8

Ansorena, Á. (1996). 15 casos para la selección de personal con éxito, Barcelona Paidós. Empresa, http://www.scielo.org.mx/pdf/eq/v20n3/ v20n3a10.pdf

Aguerrondo, I. (2000). Formación de Docentes para la Innovación Pedagógica. Argentina. Disponible en: http://www.ibe.unesco.org/regional/latin oamericannetworkpdf/maldorepar2. Pdf

AQU (2002 c.p. Cano 2005). El profesorado universitario en la sociedad del conocimiento: Competencia profesionales Docente. Universidad de Barcelona (España)

Bunk, G. (1994). La transmisión de las competencias en la formación $y$ perfeccionamiento profesionales de la RFA. Formación Profesional. Revista Europea, 8-14

Cano, E. (2005). Cómo mejorar las competencias de los docentes. Barcelona: Graó. https://dialnet.unirioja.es/servlet/articulo? codigo $=5297165$
Delors, J. (1997). La educación encierra un tesoro. México: UNESCO

Ducci, M. (1997). El Enfoque de competencia laboral en la perspectiva internacional: formación basada en competencia laboral. Montevideo: CINTERFOR/ OIT, 1997. https://www.redalyc.org/pdf/1815/18152 1554001.pdf

Farías, J. (2008). Formación Docente en Venezuela: reflexión y visión crítica. Revista Investigación y Educación. Año 5, Número 9: 63-73. UPEL

Frade, L. (2009). Desarrollo de competencias en educación: desde preescolar hasta el bachillerato. México, DF: Inteligencia Educativa

Hoffman, T. (1999). The meanings of competency. Journal of European Industrial Training. 23 (6), 275-285

Leboyer, L. (2003). Perfiles docentes para el espacio europeo de educación superior (EEES) en el ámbito universitario español. https://www.uv.es/RELIEVE/v15n1/RELIE VEv15n1_1.pdf

Ley Orgánica de Educación (2009). Gaceta oficial Numero 592915 de agosto de 2009

Ley de Universidades (1970). Gaceta oficial de la República de Venezuela Extraordinaria número 1429 de fecha 08 de septiembre de 1970

Luque, M. y Mujica de López (2006). Prácticas Profesionales Docentes: Eje Integrador entre la Universidad y la Escuela. Caso: Núcleo LUZ Punto Fijo. . Revista de educación. [Revista en Línea]. Disponible: http://www.revistas.luz.edu.ve/index.php/ $\mathrm{mc} /$ article/viewFile/112/94

Luthans, F. y Youssef, C. (2004). Human, Social, and Now Positive Psychological Capital Management: Investing in People for Competitive Advantage. Organizational Dynamics, Vol. 33, No. 2, pp. 143-160, 2004

Reglamento de los Institutos y Colegios Universitarios (1995). Decreto 865: Gaceta 4995: Gaceta. 31-Octubre-1995. https://es.calameo.com/read/003906 506c704c1cab0b7 
Ministerio del Poder Popular de Ciencias y Tecnología (MPPCT /2015). Disponible en www.mppeuct.gob.ve

Miyahira, J. (2007). La sociedad del conocimiento: un nuevo reto. Revista médica Herediana. Knowledge society: A new challenge

http://www.scielo.org.pe/scielo.php?script=sc i_arttext\&pid=S1018130X2007000100001

Morin, E. (2000). Los siete saberes necesarios para la educación del futuro. Organización de las Naciones Unidas para la Educación, la Ciencia y la Cultura. http://www.ideassonline.org/public/pdf/ LosSieteSaberesNecesariosParaLaEdudelF uturo.pdf

Morín, E. (1999). La Cabeza bien Puesta. Repensar la Reforma, Reformar el Pensamiento. Editorial Nueva Visión SAIC. Argentina

Mclelland, D. C. (1973). Testing for competence rather than for intelligence. American Psychologist, 28(1), 1-14. Cambridge: Cambridge University Press

Mertens, L. (1996). Competencia laboral: sistemas, surgimiento y modelos. Montevideo: Cinterfor

Pavie, A. (2009). Caracterización de la formación inicial del Docente. Source: Revista LIDER. Jul-dic 2012, Vol. 21, p199-219

Perrenoud, P. (2004): Diez nuevas competencias para enseñar. Barcelona: Graó

Ropé, F. y Tanguy, L. (1994). Savoirs et compétences. De l'usase de ces notions dans l'école et l'entreprise. L'HarmattanLogiques Sociales, París

Scriven, M. (1997). Modelo de evaluación. En: Castellanos Quintero S. Apuntes bibliográficos para una evaluación de programas educativos y educación comparada. Riobamba: CDES

Sen, A., Zamagni S. y Scazzieri, R. (2008). Markets, Money and Capital. Eds: Scazzieri, R. \& Zamagni, S. Cambridge: Cambridge University Press

Tobón, S. (2008). Gestión curricular y ciclos propedéuticos. Bogotá: ECOE

Tobón, S. (2006). Formación basada en competencias. Pensamiento complejo, diseño curricular y didáctica. Bogotá: Ecoe Ediciones

Trías, D. (2008). Enseñanza de la autorregulación del aprendizaje en Filosofía. Flacso. Universidad Autónoma de Madrid. http://repositorio.flacsoandes. edu.ec:8080/bitstream/10469/3402/2/TF LACSO-2009DTS.pdf

UNESCO (2000). Desafíos de la Educación. Diez módulos destinados a los responsables de los procesos de transformación educativa. Buenos Aires

Uricare A, (2010). Apuntes y perspectivas hacia otra formación docente inicial. file://C:/Users/MIGDAL 1/AppData/Loc al/Temp/5119-12359-1-PB-3.pdf

Segura, M. (2004). Competencias personales del docente. file:///C:/Users/Migdalia\%20 Perozo/Documents/5-2611\%20COMP\%20PERSONALES\%20DEL\% 20DOC\%20M\%20SEGURA.pdf

Villarroel, C. (1998). La importancia de la autorregulación en el proceso de evaluación. Reflexiones para el caso Venezuela, Cuadernos del CENDES

Zabala, A., y Arnau, L. (2008). 11 ideas clave. El aprendizaje y la enseñanza por competencias. Madrid, España: Graó 